\title{
Level set method with topological derivatives in shape optimization
}

\author{
Piotr FULMANSKI $\dagger$, Antoine LAURAIN $\ddagger$, Jean-François SCHEID $\ddagger$, Jan \\ SOKOŁOWSKI $\ddagger$
}

$\dagger$ Universiti of Łódź, Faculty of Mathematics

Banacha 22, 90-232 Łódź, Poland,

fulmanp@imul.math.uni.lodz.pl

$\ddagger$ Institut Elie Cartan UMR 7502, Nancy-Université, CNRS, INRIA, B.P. 239,

54506 Vandoeuvre-lès-Nancy Cedex, France,

antoine.laurain@iecn.u-nancy.fr,

jean-francois.scheid@iecn-u.nancy.fr,

sokolows@iecn.u-nancy.fr

\begin{abstract}
A class of shape optimization problems is solved numerically by the level set method combined with the topological derivatives for topology optimization. Actually, the topology variations are introduced on the basis of asymptotic analysis, by an evaluation of extremal points (local maxima for the specific problem) of the socalled topological derivatives introduced by Sokolowski and Zochowski [24] for elliptic boundary value problems. Topological derivatives are given for energy functionals of linear boundary value problems. We present results, including numerical examples, which confirm that the application of topological derivatives in the framework of the level set method really improves the efficiency of the method. Examples show, that the level set method combined with the asymptotic analysis, is robust for the shape optimization problems, and it allows to identify the better solution compared to the pure level set method exclusively based on the boundary variation technique.
\end{abstract}

Key Words. Shape optimization, level set method, topological derivative, optimal design.

\section{Introduction}

Shape optimization for elliptic equations is an important branch of calculus of variations, with numerous applications in structural mechanics and to solution of inverse problems. The existence of solutions for shape optimization problems can be assured under mild conditions, e.g. under the so-called Mosco convergence of the Sobolev spaces associated with the minimizing sequence of geometrical domains, however optimality conditions require more regularity of boundaries of admissible domains. Recently, a particular attention is paid to numerical methods which during the optimization process allow for the topology changes of geometrical domains. One of the candidates for such framework is the level set function which models the domain evolution and can be determined by solving appropriate Hamilton-Jacobi equations. However, by its nature, the obtained level set function decreases the number of connected components of resulting geometrical domains during the optimization process. To improve the performance of the method based on the level set function, asymptotic analysis is employed, and location of additional holes in geometrical 
domains is determined by analysis of the so-called topological derivatives of shape functionals under study. In the paper we describe in details such a combination, used already in literature, and provide some examples which show that the method is robust, in particular improves the optimal value of the shape functional obtained as a result of computations. For the convenience of the reader we present as well the elements of mathematical analysis including asymptotic analysis of specific boundary value problems with respect to small parameter which models singular perturbations of geometrical domains. Such analysis is required for determination of topological derivatives.

The numerical method for shape and topology optimization of an energy functional is presented in the paper. The method combines the shape gradient technique and the asymptotic analysis for maximization of an energy functional for elliptic equations. The level set method is used for the evolution of geometrical domains with the moving boundaries determined from the shape sensitivity analysis of the energy shape functional, the topology changes are performed in addition by analysis of the topological derivatives. It is still an open problem to devise how the combination of boundary variations and singular perturbations of geometrical domains enters in a general framework of shape optimization. One possibility is given by the so-called self-adjoint extensions of differential operators, where the small hole is approximated by singular perturbation of the coefficients of differential operator. We refer the reader to [18], [11], [14], [16], [17], for some results in this direction. We consider only steady state boundary value problems, however the same results can be obtained for the evolution problems described by parabolic and hyperbolic pde's.

The Hamilton-Jacobi nonlinear hyperbolic equation models the evolution of the level set function. The normal speed of the moving boundaries are determined from the shape gradients obtained for the energy functional [29]. Since the shape gradients serve as the coefficients for the hyperbolic equation, the standard assumption is to assume that the shape gradients are given by restriction to the moving boundaries of given functions defined in whole geometrical domains, which is unfortunately very restrictive assumption, however it is satisfied in our case.

During the numerical process, the topology changes are defined by analysis of the local maxima of topological derivatives for the energy functional. In this way, small holes can be included into the actual geometrical domain with the centers at the points determined by maximization of the topological derivatives. We inject only one hole at each etap of the method, however the asymptotic analysis furnish the information which allows for the injection of a finite number of holes, if necessary and it turns out to be useful. The size of the holes can be determined by the second order topological derivatives and this technique is not employed in the present paper.

The paper is selfcontained, thus, in appendix we provide also some technical results which are used in order to determine the topological derivatives. To this end the domain decomposition technique is applied and the proof of the asymptotic expansion for the related Steklov-Poincaré operator is given. Such an approach for evaluation of topological derivatives for energy type functionals is proposed e.g., in [12] and [27].

Finally, we present a list of references on the derivation and application of the asymptotic analysis in shape optimization. Singular perturbations of domains in the framework of shape optimization are studied by many authors, including [1], [9], [10], [13], [14], [15], [16], [17], [18], [19], [24], [25], [26]. The construction of the asymptotic expansion for the Steklov-Poincaré operator is given in [28]. Numerical results for shape and topology optimization are presented in section 9.

\section{The linear problem}

We present the numerical method for a scalar elliptic boundary value problems, and for the energy type shape functional. For such problem we can provide complete analysis on shape differentiation and asymptotic expansions of solutions with respect to small parameter which measures the singular perturbations of geometrical domain. For the sake of simplicity we restrict 
ourselves to two spatial dimensions, and to the holes in the form of a circle. In such a case by Fourier analysis we can obtain the explicit solutions for auxiliary problems in rings. Otherwise, for any spatial dimensions and arbitrary shape of holes the analysis is based on the fundamental solutions for differential operators and can be performed in the same way, we refer the reader e.g., to [18] for a general approach to the derivation of topological derivatives for elliptic boundary value problems in arbitrary spatial dimensions.

To begin with, we introduce the model problem. Let $U$ and $V$ be two bounded open subsets of $\mathbb{R}^{2}$ such that $V \subset \subset U$. For any open set $\omega \subset \mathbb{R}^{2}$, we denote by $\# \bar{\omega}$ the number of connected components of $\bar{\omega}$ and we consider the set of admissible domains

$$
\mathcal{O}_{k}=\{\Omega=U \backslash \bar{\omega} ; \omega \text { open set, } \omega \subset V, \# \bar{\omega} \leq k\} .
$$

For any $\Omega \in \mathcal{O}_{k}, k \geq 1$, the boundary of $\Omega$ can be splitted into $\partial \Omega=\Gamma_{N} \cup \partial U$ with $\Gamma_{N}=\partial \omega$. The boundary $\partial U$ is also denoted by $\Gamma_{D}$. The boundary $\Gamma_{N}$ receives Neumann boundary conditions, while we have Dirichlet conditions on $\Gamma_{D}$. Let us point out that the open set $\omega$ is not necessarily any connected set as illustrated in Figure 1.

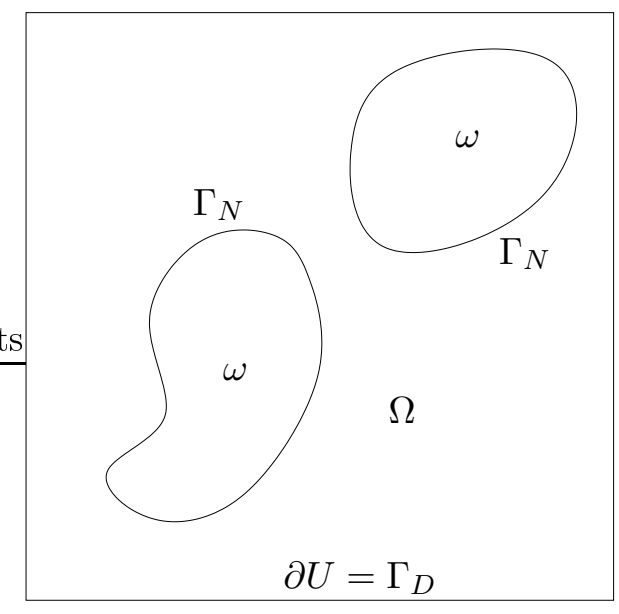

Fig. 1 - An admissible domain $\Omega$

For $f \in C^{\infty}(\bar{U})$, let us consider the following problem

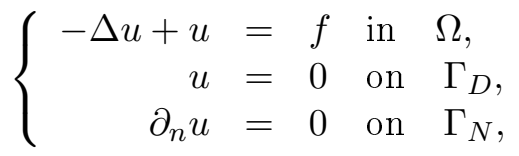

where $n$ is the unit outwards normal vector to $\partial \Omega$, and $\partial_{n}$ stands for the normal derivative on $\partial \Omega$. In addition, let us consider the energy functional depending on the solution $u$ to (2) :

$$
E(\Omega, u):=\frac{1}{2} \int_{\Omega}\left(|\nabla u|^{2}+u^{2}\right) d x-\int_{\Omega} f u d x=-\frac{1}{2} \int_{\Omega}\left(|\nabla u|^{2}+u^{2}\right) d x=-\frac{1}{2} \int_{\Omega} f u d x,
$$

and let us introduce the regularized shape functional

$$
J(\Omega):=E(\Omega, u)+\lambda A(\Omega)-\mu P_{c}(\Omega)^{2},
$$

with $A(\Omega)$ and $P_{c}(\Omega)$ defined by

$$
\begin{aligned}
A(\Omega) & :=|\Omega|, \\
P_{c}(\Omega) & :=\max (0, \ell(\partial \Omega)-c) .
\end{aligned}
$$

In the above definitions, $|\Omega|$ denotes the Lebesgue measure of $\Omega$ in $\mathbb{R}^{2}$ and $\ell(\partial \Omega)$ is the 1 dimensional measure of $\partial \Omega$. Constants $\lambda$ and $\mu$ are positive and allow us to take into account 
the area and perimeter constraints respectively. The constant $c$ is also positive and corresponds to a shifted perimeter functional, i.e. beyond $c$, the perimeter constraint becomes active.

For any fixed integer $k \geq 1$, let us consider the following shape optimization problem

$$
\max \left\{J(\Omega): \Omega \in \mathcal{O}_{k}\right\} .
$$

Since the exterior boundary of $\Omega$ is fixed once for all and equals to $\partial U$, the domains $\Omega$ satisfying (7) are actually determined by their moving boundaries $\Gamma_{N}$.

In the following three sections, we perform the mathematical analysis of problem (7). We show that problem (7) is well posed in the sense that there exists an optimal domain, furthermore, the optimal shape can be characterized by appropriate necessary optimality conditions. For a given topology of an optimal domain there are two different sort of optimality conditions. On the moving boundaries, the shape gradients can be evaluated and the optimality conditions say that the optimal value of the cost cannot be improved by admissible boundary variations. On the other hand, the sign of topological derivatives is fixed inside of the geometrical domain, in such a way that any creation of a small hole cannot improve the value of the cost. The second condition is very strong, it seems, therefore it is a very difficult task to find an optimal domain which satisfies such a condition.

Actually, we study the existence of an optimal domain in section 3, and then the shape sensitivity in section 4, the topological derivatives of the functional $J$ are obtained in section 5 . The construction of a level set representation is based on the shape derivatives of $J$, as it is described in section 6 . This formulation provides a practical way to increase the values of the shape functional $J$. The appearance of new holes is not possible with the only use of the level set method based on the shape derivatives. Therefore, an application of topological derivative allows us to create new holes in order to increase the values of $J$.

\section{Existence of an optimal domain}

It can be showed that there exists a domain $\Omega$, solution of (7). We make use of a result from Bucur and Varchon [3] and [4] for the Neumann boundary conditions on the holes. Similar result for the Dirichlet boundary conditions in two spatial dimensions is given by Sverak [30]. With a few changes in the proof of this result, we can apply the results from [3] and [4] to our problem. It is possible without any additional difficulty since the boundary $\Gamma_{D}$ is fixed. Thus, we obtain the following theorem

Theorem 1 Let $\Omega_{i}=U \backslash \bar{\omega}_{i}$ be a sequence of open sets such that the number of connected components of $\bar{\omega}_{i}$ is uniformly bounded. If $\bar{\omega}_{i}$ converges in the sense of the Hausdorff metric to $\bar{\omega}$, then the solution $u_{i}$ of the Neumann problem

$$
\left\{\begin{array}{rll}
-\Delta u_{i}+u_{i}= & f & \text { in } \Omega_{i} \\
u_{i} & =0 & \text { on } \partial U \\
\partial_{n} u_{i}= & 0 & \text { on } \partial \omega_{i} .
\end{array}\right.
$$

converges to the solution $u$ of problem (8) on $\Omega$ if and only if $\left|\Omega_{i}\right| \rightarrow|\Omega|$. (All functions are implicitely extended to zero by setting $u_{i}=0$ in $\omega_{i}$ and $u=0$ in $\omega$ and the convergence takes place in $\left.L^{2}(U)\right)$.

Now it is possible to prove the existence of an optimal domain.

Theorem 2 For any $k \geq 0$, Problem (7) admits at least one solution $\Omega \in \mathcal{O}_{k}$.

Proof. Let $\left\{\Omega_{i}\right\}, i=0,1,2, \cdots$, be a maximizing sequence for Problem (7). There exists a subsequence still denoted by $\Omega_{i}$ such that $\Omega_{i} \rightarrow \Omega$ for $i \rightarrow+\infty$, in the sense of Hausdorff metric. In what follows, such a convergence is denoted by $\Omega_{i} \stackrel{H}{\longrightarrow} \Omega$. For the definition of Hausdorff 
convergence, see [8].

It is shown in [8] that the number of connected components of $\Omega^{c}$ is bounded and we also have by taking the limit

$$
\ell(\partial \Omega) \leq \liminf _{i} \ell\left(\partial \Omega_{i}\right)
$$

Thus $\ell(\partial \Omega)<\infty$ since we maximize $J(\Omega)$ and thus $P_{c}(\Omega)$ is bounded. Using the notion of density perimeter introduced in [2], it is well known that the Hausdorff convergence of $\Omega_{i}$ to $\Omega$ implies convergence in the sense of characteristic functions :

$$
\Omega_{i} \stackrel{H}{\longrightarrow} \Omega \Longrightarrow \chi_{\Omega_{i}} \stackrel{L^{1}(U)}{\longrightarrow} \chi_{\Omega} .
$$

As a consequence, we get for $i \rightarrow+\infty$, the convergence of Lebesgue measures

$$
\left|\Omega_{i}\right| \rightarrow|\Omega| \text {. }
$$

To conclude, we have to prove the convergence of $E\left(\Omega_{i}, u_{i}\right)=-\frac{1}{2} \int_{\Omega_{i}} f u_{i}$. Moreover, we have

$$
\begin{aligned}
\left|\int_{\Omega_{i}} f u_{i}-\int_{\Omega} f u\right| & =\left|\int_{U} f u_{i} \chi_{\Omega_{i}}-\int_{U} f u \chi_{\Omega}\right| \\
& =\left|\int_{U} f u_{i}\left(\chi_{\Omega_{i}}-\chi_{\Omega}\right)+\int_{U} f \chi_{\Omega}\left(u_{i}-u\right)\right| \\
& \leq\left\|u_{i}\right\|_{2}\left\|f\left(\chi_{\Omega_{i}}-\chi_{\Omega}\right)\right\|_{2}+\left\|f \chi_{\Omega}\right\|_{2}\left\|u_{i}-u\right\|_{2},
\end{aligned}
$$

where $\|\cdot\|_{2}$ is the norm in $L^{2}(U)$. According to Theorem 1 , we have

$$
\left\|u_{i}\right\|_{2} \rightarrow\|u\|_{2} \quad \text { and } \quad\left\|u_{i}-u\right\|_{2} \rightarrow 0 .
$$

Furthermore,

$$
\left\|f\left(\chi_{\Omega_{i}}-\chi_{\Omega}\right)\right\|_{2} \rightarrow 0
$$

thanks to the convergence in the sense of characteristic functions of $\chi_{\Omega_{i}}$ to $\chi_{\Omega}$. Thus, we get

$$
E\left(\Omega_{i}\right) \rightarrow E(\Omega)
$$

Finally, combining convergences (14), (11) and (9), we obtain the inequality

$$
J(\Omega) \geq \limsup _{i} J\left(\Omega_{i}\right) .
$$

Thus, $\Omega$ is a solution to problem (7).

Remark 1 In order to use the shape derivatives, we need the domains with smooth boundaries. The solutions of (7) are not necessarily smooth. From now on, we assume the existence of an optimal domain for (7) which in addition, is smooth enough.

\section{Shape derivatives}

Level set method requires the knowledge of shape gradients for the functional to be maximized. Let $\Omega$ be a sufficiently regular domain. The variational formulation of (2) is given by

$$
u \in H_{\Gamma_{D}}^{1}(\Omega): \int_{\Omega}\langle\nabla u, \nabla w\rangle d x+\int_{\Omega} u w d x=\int_{\Omega} f w d x \quad \forall w \in H_{\Gamma_{D}}^{1}(\Omega),
$$

where $H_{\Gamma_{D}}^{1}(\Omega)=\left\{v \in H^{1}(\Omega) \mid v=0\right.$ on $\left.\Gamma_{D}\right\}$. 
We describe the construction of regular perurbations of $\Omega$, which is used to obtain the shape gradients for shape functionals [5],[29]. Let $\xi$ be a vector field of class $C^{k}$ with compact support in $U$. We put $|\xi|=\|\xi\|_{C^{k}\left(U ; \mathbb{R}^{2}\right)}$. Let us consider the transformation $F_{\xi}=I+\xi$ and define $\Omega_{\xi}=F_{\xi}(\Omega)$. We assume that $|\xi|$ is small enough, so that $F_{\xi}$ is a $C^{k}$-diffeomorphism. As a consequence, there exists a unique solution $u^{\xi} \in H_{\Gamma_{D}}^{1}\left(\Omega_{\xi}\right)$ to the variational equation in variable domain $\Omega_{\xi}$,

$$
\int_{\Omega_{\xi}}\left\langle\nabla u^{\xi}, \nabla v\right\rangle d x+\int_{\Omega_{\xi}} u^{\xi} v d x=\int_{\Omega_{\xi}} f v d x \quad \forall v \in H_{\Gamma_{D}}^{1}\left(\Omega_{\xi}\right) .
$$

We are going to show that the energy functional $E(\Omega, u)$ is differentiable with respect to the domain and we compute its shape derivative $d E(\Omega, \xi)$ defined for any direction $\zeta=\xi /|\xi|$ of the unit norm $|\zeta|=1$ by

$$
d E(\Omega, \xi):=\lim _{t \rightarrow 0} \frac{E\left(\Omega_{\xi}, u^{\xi}\right)-E(\Omega, u)}{t},
$$

where $t=|\xi|$, and for the sake of simplicity, in the notation we write $\xi$ instead of $\zeta$.

We need to transform (17) on the fixed domain $\Omega$. To this end, we denote $u_{\xi}$ the transported function defined by the composition

$$
u_{\xi}=u^{\xi} \circ F_{\xi} \in H_{\Gamma_{D}}^{1}(\Omega) .
$$

Using the change of variable in (17), we obtain the variational equation satisfied by $u_{\xi}$ :

$$
\int_{\Omega}\left\langle\left(D F_{\xi}^{T}\right)^{-1} \nabla u_{\xi},\left(D F_{\xi}^{T}\right)^{-1} \nabla w\right\rangle q_{\xi} d x+\int_{\Omega} u_{\xi} w q_{\xi} d x=\int_{\Omega} f_{\xi} w q_{\xi} d x \quad \forall w \in H_{\Gamma_{D}}^{1}(\Omega),
$$

where $f_{\xi}=f \circ F_{\xi}$. In the above formulation, $D F_{\xi}$ is the Jacobian matrix of the transformation $F_{\xi}$ and $q_{\xi}$ stands for the determinant of the Jacobian. More precisely, we have

$$
\begin{aligned}
D F_{\xi} & =I+D \xi \\
q_{\xi} & =\operatorname{det} D F_{\xi}=1+\operatorname{div} \xi+\operatorname{det} D \xi
\end{aligned}
$$

It is shown in [6] that $u_{\xi}$ admits the following Taylor expansion

$$
u_{\xi}=u+u^{1}(\xi)+\tilde{u}(\xi),
$$

where the material derivative $u^{1}(\xi)$ is the unique solution of the variational equation

$$
\begin{aligned}
\int_{\Omega}\left\langle\nabla u^{1}(\xi), \nabla w\right\rangle d x= & \left.\int_{\Omega}\left\langle A(\xi)^{-1}\right) \nabla u, \nabla w\right\rangle d x+\int_{\Omega} g(\xi) w d x \\
& -\int_{\Omega}\left(u w \operatorname{div}(\xi)+u^{1} w\right) d x \quad \forall w \in H_{\Gamma_{D}}^{1}(\Omega) .
\end{aligned}
$$

The matrix $A(\xi)$ and the function $g(\xi)$ are given by

$$
\begin{gathered}
A(\xi)=D \xi+D \xi^{T}-(\operatorname{div} \xi) I, \\
g(\xi)=\operatorname{div}(f \xi) .
\end{gathered}
$$

Expansion (22) holds true with the estimates (see [6])

$$
\begin{aligned}
\left\|u_{\xi}-u\right\|_{H_{\Gamma_{D}}^{1}(\Omega)} & \leq c|\xi|, \\
\|\tilde{u}(\xi)\|_{H_{\Gamma_{D}}^{1}(\Omega)}=\left\|u_{\xi}-u-u^{1}(\xi)\right\|_{H_{\Gamma_{D}}^{1}(\Omega)} & \leq c|\xi|^{2} .
\end{aligned}
$$


Making use of the change of variables (18) in the expression $E\left(\Omega_{\xi}, u^{\xi}\right)=-\frac{1}{2} \int_{\Omega_{\xi}}\left(\left|\nabla u^{\xi}\right|^{2}+\left(u^{\xi}\right)^{2}\right) d x$ together with the estimates (24) and (25), we obtain that $E$ is differentiable with respect to the domain and its first order shape derivative is given by

$$
\begin{aligned}
d E(\Omega ; \xi)= & -\int_{\Omega}\left\langle\nabla u, \nabla u^{1}(\xi)\right\rangle d x+\frac{1}{2} \int_{\Omega}\langle A(\xi) \nabla u, \nabla u\rangle d x \\
& -\int_{\Omega} u u^{1}(\xi) d x-\frac{1}{2} \int_{\Omega} u^{2} \operatorname{div}(\xi) d x .
\end{aligned}
$$

Now, choosing $w=u$ in the variational identity (23), we obtain

$$
\int_{\Omega}\left\langle\nabla u, \nabla u^{1}(\xi)\right\rangle d x=\int_{\Omega}\langle A(\xi) \nabla u, \nabla u\rangle d x+\int_{\Omega} g(\xi) u d x-\int_{\Omega}\left(u^{2} \operatorname{div}(\xi)+u^{1}(\xi) u\right) d x .
$$

In view of $(27)$, we can get rid of $u^{1}(\xi)$ in $(26)$ :

$$
d E(\Omega ; \xi)=-\frac{1}{2} \int_{\Omega}\langle A(\xi) \nabla u, \nabla u\rangle d x-\int_{\Omega} g(\xi) u d x+\frac{1}{2} \int_{\Omega} u^{2} \operatorname{div}(\xi) d x .
$$

Starting from this expression, we can obtain a more useful expression for the shape derivative. First of all, we turn to the first integral term in the right hand side of (28). We have

$$
\begin{aligned}
\frac{1}{2} \int_{\Omega}\langle A(\xi) \nabla u, \nabla u\rangle d x & =\int_{\Omega}\langle D \xi \nabla u, \nabla u\rangle d x-\frac{1}{2} \int_{\Omega}|\nabla u|^{2} \operatorname{div}(\xi) d x \\
& =\int_{\Omega}\langle\nabla(\langle\xi, \nabla u\rangle), \nabla u\rangle d x-\int_{\Omega}\left\langle D^{2} u \xi, \nabla u\right\rangle d x-\frac{1}{2} \int_{\Omega}|\nabla u|^{2} \operatorname{div}(\xi) d x \\
& =-\int_{\Omega} \Delta u\langle\xi, \nabla u\rangle d x+\int_{\partial \Omega}\langle\xi, \nabla u\rangle\langle\nabla u, n\rangle d \sigma-\int_{\Omega}\left\langle D^{2} u \xi, \nabla u\right\rangle d x \\
& =-\frac{1}{2} \int_{\Omega}|\nabla u|^{2} \operatorname{div}(\xi) d x
\end{aligned}
$$

Since $-\Delta u=f-u$ in $\Omega$, equation (28) becomes

$$
\begin{aligned}
d E(\Omega ; \xi)= & -\int_{\Omega} f\langle\xi, \nabla u\rangle d x-\int_{\Omega} \operatorname{div}(f \xi) u d x \\
& +\int_{\Omega}\left\langle D^{2} u \xi, \nabla u\right\rangle d x+\frac{1}{2} \int_{\Omega}|\nabla u|^{2} \operatorname{div}(\xi) d x \\
& +\int_{\Omega} u\langle\xi, \nabla u\rangle d x+\frac{1}{2} \int_{\Omega} u^{2} \operatorname{div}(\xi) d x \\
& -\int_{\partial \Omega}\langle\xi, \nabla u\rangle\langle\nabla u, n\rangle d \sigma .
\end{aligned}
$$

Finally we obtain

$$
d E(\Omega ; \xi)=\int_{\Omega} \operatorname{div}\left(\frac{1}{2}|\nabla u|^{2} \xi+\frac{1}{2} u^{2} \xi-f \xi u\right) d x-\int_{\partial \Omega}\langle\xi, \nabla u\rangle\langle\nabla u, n\rangle d \sigma .
$$

Since $\langle\nabla u, n\rangle=0$ on $\Gamma_{N}$ and $\xi$ has compact support in $U$, we have

$$
d E(\Omega ; \xi)=\int_{\Gamma_{N}}\left(\frac{1}{2}|\nabla u|^{2}+\frac{1}{2} u^{2}-f u\right)\langle\xi, n\rangle d \sigma .
$$

Moreover, the shape derivatives of $A(\Omega)$ and $P_{c}(\Omega)$ are given by

$$
\begin{aligned}
d A(\Omega ; \xi) & =\int_{\Gamma_{N}}\langle\xi, n\rangle d \sigma \\
d P_{c}(\Omega ; \xi) & =\mathbb{1}_{\{\ell(\partial \Omega)=c\}} \max \left(0, \int_{\Gamma_{N}} \mathcal{H}\langle\xi, n\rangle d \sigma\right)+\mathbb{1}_{\left\{P_{c}(\Omega)>0\right\}} \int_{\Gamma_{N}} \mathcal{H}\langle\xi, n\rangle d \sigma \\
d P_{c}(\Omega ; \xi)^{2} & =2 P_{c}(\Omega) \int_{\Gamma_{N}} \mathcal{H}\langle\xi, n\rangle d \sigma
\end{aligned}
$$


where $\mathcal{H}$ is the curvature of $\Gamma_{N}$. Thus, the shape derivative $d J(\Omega ; \xi)$ of $J(\Omega)$ is given by

$$
d J(\Omega ; \xi)=\int_{\Gamma_{N}}\left(\frac{1}{2}|\nabla u|^{2}+\frac{1}{2} u^{2}-f u+\lambda-2 \mu P_{c}(\Omega) \mathcal{H}\right)\langle\xi, n\rangle d \sigma
$$

\section{Topological derivative}

For the sake of simplicity, we assume in this section that $\Omega=U$ (i.e. $\Gamma_{N}=\emptyset$ ). Let us now consider the perforated domain $\Omega_{\rho}=U \backslash \bar{B}_{\rho}$ where $B_{\rho}$ is the ball of radius $\rho$, centered at a point $x_{0} \in U$ and with boundary $\Gamma_{\rho}=\partial B_{\rho}$. In order to study the topological derivative of the functional $J$ for the linear problem (2), we need to compute the asymptotic expansion of the energy (see (3))

$$
E\left(\Omega_{\rho}, u_{\rho}\right)=-\frac{1}{2} \int_{\Omega}\left(\left|\nabla u_{\rho}\right|^{2}+u_{\rho}^{2}\right) d x
$$

where $u_{\rho}$ is the solution of the linear problem

$$
\left\{\begin{aligned}
-\Delta u_{\rho}+u_{\rho}=f & \text { in } \Omega_{\rho}, \\
u_{\rho}=0 & \text { on } \partial U=\Gamma_{D}, \\
\partial_{n} u_{\rho}=0 & \text { on } \Gamma_{\rho} .
\end{aligned}\right.
$$

In order to study the topological derivative, we used the so-called truncated domain technique (see [27], [12]). We only present the main sketch of the method used to compute the asymptotic expansion of $J$ and we refer to the appendix for a complete proof of the results. We turn to the truncated domain technique by considering the domain

$$
\Omega_{R}=U \backslash \bar{B}_{R},
$$

where $B_{R}$ is the ball of radius $R>\rho$, centered at $x_{0}$. We also define the ring $C(R, \rho)$ such that $\Omega_{\rho}=\Omega_{R} \cup \Gamma_{R} \cup C(R, \rho)$ where $\Gamma_{R}$ denotes the boundary of the ball $B_{R}$ i.e. $\Gamma_{R}=\partial B_{R}$. (see Figure 2).

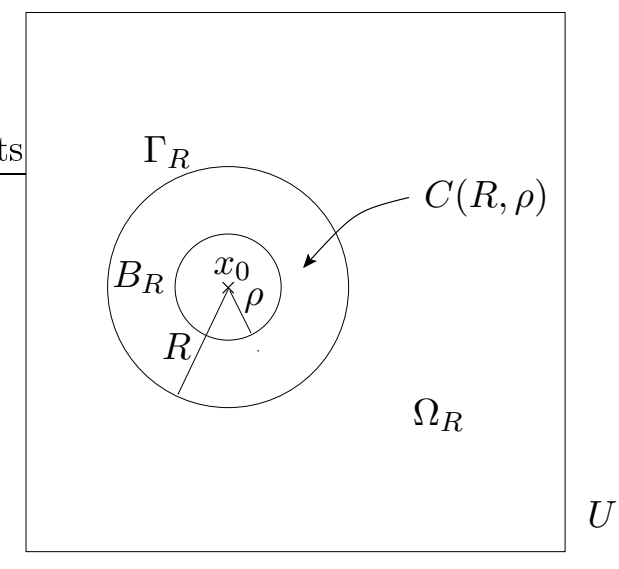

FIG. 2 - The truncated domain technique.

Then we consider the following truncated problem

$$
\left\{\begin{aligned}
-\Delta u_{\rho}^{R}+u_{\rho}^{R}=f & \text { in } \Omega_{R}, \\
u_{\rho}^{R}=0 & \text { on } \partial U \\
-\partial_{n} y_{\rho}+\partial_{n} u_{\rho}^{R}=A_{\rho}\left(u_{\rho}^{R}\right) & \text { on } \Gamma_{R} .
\end{aligned}\right.
$$

In the above problem, $A_{\rho}$ is the Stekov-Poincare operator defined for any $v \in H^{\frac{1}{2}}\left(\Gamma_{R}\right)$ by

$$
A_{\rho}(v)=\partial_{n} w_{\rho}
$$


where $w_{\rho}=w_{\rho}(v)$ is the unique solution of the problem

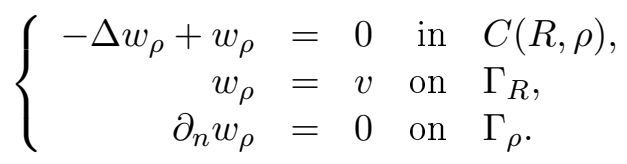

Finally, the function $y_{\rho}$ appearing in problem (37) is the solution of the following problem

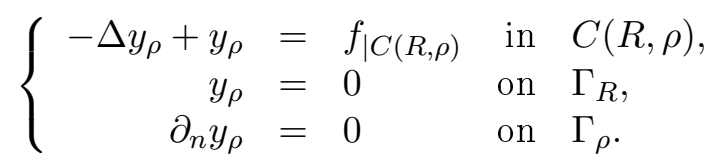

Then, it can be easily shown that

$$
u_{\rho}^{R}=u_{\rho \mid \Omega_{R}} \quad \text { and } \quad u_{\rho \mid C(R, \rho)}=w_{\rho}\left(u_{\rho}^{R}\right)+y_{\rho} .
$$

In order to obtain the topological derivative of $J$, we have to perform an expansion of the energy functional $E\left(\Omega_{\rho}, u_{\rho}\right)=-\frac{1}{2} \int_{\Omega_{\rho}}\left(\left|\nabla u_{\rho}\right|^{2}+u_{\rho}^{2}\right) d x$ with respect to the radius $\rho$. Using the domain truncation, we can split the integral in two parts, and we obtain

$$
E\left(\Omega_{\rho}, u_{\rho}\right)=E\left(\Omega_{R}, u_{\rho}^{R}\right)-\frac{1}{2} E_{\rho}^{(1)}\left(u_{\rho}^{R}\right)+\frac{1}{2} E_{\rho}^{(2)}(f)
$$

where

$$
E\left(\Omega_{R}, u_{\rho}^{R}\right)=-\frac{1}{2} \int_{\Omega_{R}}\left(\left|\nabla u_{\rho}^{R}\right|^{2}+\left(u_{\rho}^{R}\right)^{2}\right) d x
$$

and

$$
\begin{aligned}
E_{\rho}^{(1)}\left(u_{\rho}^{R}\right) & =\int_{C(R, \rho)}\left(\left|\nabla w_{\rho}\right|^{2}+w_{\rho}^{2}\right) d x \quad \text { with } \quad w_{\rho}=w_{\rho}\left(u_{\rho}^{R}\right), \\
E_{\rho}^{(2)}(f) & =-\int_{C(R, \rho)}\left(\left|\nabla y_{\rho}\right|^{2}+y_{\rho}^{2}\right) d x .
\end{aligned}
$$

Studying problems (37), (39) and (40), we can obtain asymptotic expansion of (43), (44) and (45) respectively which lead to the following expansion for the energy (see the appendix)

$$
E\left(\Omega_{\rho}, u_{\rho}\right)=E(\Omega, u)-\left(\frac{u\left(x_{0}\right)^{2}}{2}+\left|\nabla u\left(x_{0}\right)\right|^{2}-f\left(x_{0}\right) u\left(x_{0}\right)\right) \pi \rho^{2}+o\left(\rho^{2}\right) .
$$

Now, using the expansions

$$
\begin{aligned}
A\left(\Omega_{\rho}\right) & =A(\Omega)-\pi \rho^{2}, \\
P_{c}\left(\Omega_{\rho}\right)^{2} & =P_{c}(\Omega)^{2}+4 \pi P_{c}(\Omega) \rho+o\left(\rho^{2}\right),
\end{aligned}
$$

we obtain the following asymptotic expansion for $J$ :

$$
J\left(\Omega_{\rho}\right)=J(\Omega)+h(\rho) \mathcal{T}_{\Omega}\left(x_{0}\right)+o(h(\rho)),
$$

with

$$
h(\rho)= \begin{cases}\pi \rho^{2} & \text { if } P_{c}(\Omega)=0, \\ \pi \rho & \text { if } P_{c}(\Omega)>0 .\end{cases}
$$

The topological derivative $\mathcal{T}_{\Omega}\left(x_{0}\right)$ of the functional $J$ at point $x_{0} \in \Omega$ is given by

- $\mathcal{T}_{\Omega}\left(x_{0}\right)=-\left|\nabla u\left(x_{0}\right)\right|^{2}-\frac{1}{2} u\left(x_{0}\right)^{2}+f\left(x_{0}\right) u\left(x_{0}\right)-\lambda, \quad$ if $P_{c}(\Omega)=0$,

- $\mathcal{T}_{\Omega}\left(x_{0}\right)=-4 \mu P_{c}(\Omega)$, if $P_{c}(\Omega)>0$. 


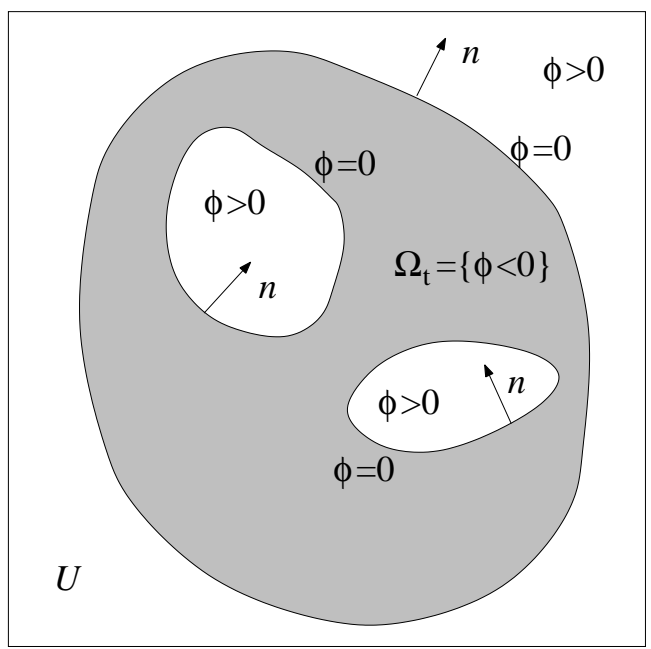

FIG. 3 - The level set representation of the domain.

\section{The level set formulation}

\subsection{The Hamilton-Jacobi equation}

In the level set method, a domain and its boundary are represented as level sets of a continuous function $\phi$. We refer to [20],[21],[23] for a complete description of the level set formulation of fronts propagating models. We give a brief description of the level set formulation for the evolution of a domain $\Omega \subset U \subset \mathbb{R}^{2}$ under a given velocity field $\xi$. Let us consider the domain $\Omega_{t}=(I+t \xi)(\Omega)$, $t \in \mathbb{R}^{+}$, with a smooth vector field $\xi$ compactly supported in $U$. For $t \geq 0$, the domain $\Omega_{t}$ and its boundary are defined by a function $\phi=\phi(x, y, t),(x, y) \in U$, such that

$$
\Omega_{t}=\{(x, y) \in U, \phi(x, y, t)<0\} \quad \text { and } \quad \partial \Omega_{t}=\{(x, y) \in U, \phi(x, y, t)=0\} .
$$

The boundary $\partial \Omega_{t}$ is the zero level curve of the function $\phi(\cdot, t)$ (see Fig. 3).

We consider the position $(x(t), y(t))$ of a particle on a (given) level curve of $\phi(\cdot, t)$ moving with velocity $\xi=\left(x^{\prime}(t), y^{\prime}(t)\right)$. Then, we have $\phi(x(t), y(t), t)=$ constant, for all $t \geq 0$. By differentiating with respect to $t$, we obtain the following transport equation

$$
\phi_{t}+\xi \cdot \nabla \phi=0 \quad \text { in } U \times \mathbb{R}^{+} .
$$

The normal unit vectors $n$ to the level sets of $\phi$ are given by $n=\nabla \phi /|\nabla \phi|$. This leads to the Hamilton-Jacobi equation

$$
\phi_{t}+\xi_{n}|\nabla \phi|=0 \quad \text { in } U \times \mathbb{R}^{+}
$$

where $\xi_{n}$ is the normal component of the velocity i.e. $\xi_{n}=\langle\xi, n\rangle$. We also have to choose initial data and boundary conditions with the Hamilton-Jacobi equation (54). The initial data $\phi(x, y, 0)=\phi_{0}(x, y)$ is chosen as the signed distance function to the initial boundary $\partial \Omega_{0}=\partial \Omega$ i.e.

$$
\phi_{0}(x, y)= \pm \operatorname{dist}\left((x, y), \partial \Omega_{0}\right) \quad \text { for }(x, y) \in U,
$$

with the minus sign (resp. plus sign) if the point $x$ is inside (resp. outside) the initial domain $\Omega_{0}=\Omega$. Finally, we choose homogeneous Neumann boundary condition on the boundary $\partial U$ of the whole domain :

$$
\partial_{n} \phi=0 \quad \text { on } \quad \partial U \times \mathbb{R}^{+} .
$$




\subsection{Normal velocity for the level set equation}

Recall that the shape derivative $d J(\Omega ; \xi)$ associated to the velocity field $\xi$ is given by (34). We locally (that is under small perturbations of the domain) require that $d J(\Omega ; \xi)>0$. This leads to the following choice for the normal component $\xi_{n}=\langle\xi, n\rangle$ of the velocity :

$$
\xi_{n}=\frac{1}{2}|\nabla u|^{2}+\frac{1}{2} u^{2}-u f+\lambda-2 \mu P_{c}(\Omega) \mathcal{H} \text { on } \Gamma_{N} .
$$

If the velocity field $\xi$ satisfies (57), we clearly have $d J(\Omega ; \xi)>0$ and then $J\left(\Omega_{t}\right)>J(\Omega)$ for $t$ small enough.

According to (57), the normal component of the velocity is only imposed along the boundary $\Gamma_{N}$. But in order to solve the Hamilton-Jacobi equation (54), we need a velocity field defined in the whole domain $U$. We write

$$
\xi_{n}=V_{n}-2 \mu P_{c}(\Omega) \mathcal{H} \text { on } \Gamma_{N}
$$

with

$$
V_{n}=\frac{1}{2}|\nabla u|^{2}+\frac{1}{2} u^{2}-u f+\lambda \text { on } \Gamma_{N} .
$$

The curvature $\mathcal{H}$ is directly linked to the level set function $\phi$ by the formula

$$
\mathcal{H}=\operatorname{div}\left(\frac{\nabla \phi}{|\nabla \phi|}\right)
$$

So, the curvature term is defined in the whole domain $U$ and the only normal velocity part $V_{n}$ given by (59) has to be extended off the boundary $\Gamma_{N}$. If $V_{\text {ext }}$ denotes the extension of $V_{n}$ to the whole domain $U$, the Hamilton-Jacobi becomes

$$
\phi_{t}+\left(V_{\text {ext }}-2 \mu P_{c}(\Omega) \operatorname{div}\left(\frac{\nabla \phi}{|\nabla \phi|}\right)\right)|\nabla \phi|=0 \quad \text { in } U \times \mathbb{R}^{+} .
$$

The construction of the extension $V_{\text {ext }}$ of $V_{n}$ to the whole domain $U$ is explained in Section 8.

\section{The shape optimization algorithm}

Now, we describe the general shape optimization algorithm.

\section{First step : initial domain}

We start to choose an initial domain $\Omega^{0}$ and we compute the solution of $(2)$ in $\Omega^{0}$ by the use of the piecewise linear finite element method. Then, the topological derivative $\mathcal{T}_{\Omega^{0}}(x)$ is computed for all $x \in \Omega^{0}$, according to (51).

\section{Second step : topological change by creating a hole}

The topological derivative allows us to find the place where a hole should be created in the domain $\Omega^{0}$ in order to increase the energy functional $J$. We find the point $x_{0} \in \Omega^{0}$ such that $\mathcal{T}_{\Omega^{0}}\left(x_{0}\right)=\max _{x \in \Omega^{0}} \mathcal{T}_{\Omega^{0}}(x)$. If $\mathcal{T}_{\Omega^{0}}\left(x_{0}\right)>0$ then a circular hole $\omega_{\rho}$ of radius $\rho>0$ and centered at $x_{0}$ is created. Then we denote by $\Omega_{\rho}^{0}$ the domain with the new hole $\omega_{\rho}$. Homogeneous Neumann condition is imposed on the boundary of the new hole. Remark that the radius of this hole should be as small as possible, depending on the space step of the mesh.

\section{Third step : shape evolution}

We turn to the evolution of the domain $\Omega_{\rho}^{0}$ with the application of the shape derivative in the level set formulation. We have to compute the solution $\phi$ to the Hamilton-Jacobi equation (61) where the initial $\phi$ is taken to be the signed distance function to the domain $\Omega_{\rho}^{0}$. We perform 
the new computation of the solution of (2) in $\Omega_{\rho}^{0}$ and then we compute the normal velocity $V_{n}$ on the internal boundary part $\Gamma_{N}$ of $\partial \Omega_{\rho}^{0}$ according to (59). The level set equation (61) require to extend the normal velocity $V_{n}$ to the whole domain $U$. This is done by solving a transport equation. The next section contains an explaination how to exactly proceed to construct the extended velocity. Only few iterations of the Hamilton-Jacobi equation are performed.

Once we have computed the level set function, we can determine the new domain $\Omega^{1}$. Then we go back top the first step of the algorithm with $\Omega^{1}$ in place of $\Omega^{0}$. We stop when convergence of the domain is reached that is when the geometrical domain does not change anymore.

\section{Numerical method for the level set equation}

In this section, we present the numerical method used to solve the Hamilton-Jacobi equation (61). We also describe the method used for computing the extension $V_{\text {ext }}$ of the normal velocity to the entire domain $U$.

\subsection{Discretization of the level set equation}

We choose $U=(0,1) \times(0,1)$ and we define the mesh grid of $U$ by introducing the nodes $P_{i j}$ with coordinates $(i \Delta x, j \Delta y)$. The parameters $\Delta x$ and $\Delta y$ are the steps discretization in the $x$ and $y$ directions, respectively. We denote by $t^{k}=k \Delta t$ the discrete time for $k \in \mathbb{N}$, where $\Delta t$ is the time step. An approximation $\phi_{i j}^{k} \simeq \phi\left(P_{i j}, t^{k}\right)$ is computed with a time explicit finite differences scheme due to Osher and Sethian [21],[23],[20]. This scheme is based on an upwind scheme for the first order part $\left(V_{n}|\nabla \phi|\right)$ of the Hamilton-Jacobi equation. For the second order part with the curvature term $(\mathcal{H}|\nabla \phi|)$, centered finite differences are used. More precisely, this scheme reads as

$$
\phi_{i j}^{k+1}=\phi_{i j}^{k}-\Delta \operatorname{tg}\left(D_{x}^{-} \phi_{i j}^{k}, D_{x}^{+} \phi_{i j}^{k}, D_{y}^{-} \phi_{i j}^{k}, D_{y}^{+} \phi_{i j}^{k}\right)
$$

where

$$
D_{x}^{-} \phi_{i j}=\frac{\phi_{i j}-\phi_{i-1, j}}{\Delta x}, \quad D_{x}^{+} \phi_{i j}=\frac{\phi_{i+1, j}-\phi_{i j}}{\Delta x}
$$

are the backward and forward approximations of the $x$-derivatives of $\phi$ at $P_{i j}$. Similar expressions hold for the approximations $D_{y}^{-}$and $D_{y}^{+}$of the $y$-derivatives. The numerical flux is given by

$$
g\left(D_{x}^{-} \phi_{i j}, D_{x}^{+} \phi_{i j}, D_{y}^{-} \phi_{i j}, D_{y}^{+} \phi_{i j}\right)=g_{i j}^{(1)}+g_{i j}^{(2)} .
$$

The numerical flux part $g_{i j}^{(1)}$ comes from the discretization of the first order part $V_{n}|\nabla \phi|$ of the Hamilton-Jacobi equation and is given by

$$
g_{i j}^{(1)}=\max \left(V_{\mathrm{ext}}\left(P_{i j}\right), 0\right) G^{+}+\min \left(V_{\mathrm{ext}}\left(P_{i j}\right), 0\right) G^{-}
$$

with

$$
\begin{aligned}
G^{+} & =\left[\max \left(D_{x}^{-} \phi_{i j}, 0\right)^{2}+\min \left(D_{x}^{+} \phi_{i j}, 0\right)^{2}+\max \left(D_{y}^{-} \phi_{i j}, 0\right)^{2}+\min \left(D_{y}^{+} \phi_{i j}, 0\right)^{2}\right]^{1 / 2} \\
G^{-} & =\left[\min \left(D_{x}^{-} \phi_{i j}, 0\right)^{2}+\max \left(D_{x}^{+} \phi_{i j}, 0\right)^{2}+\min \left(D_{y}^{-} \phi_{i j}, 0\right)^{2}+\max \left(D_{y}^{+} \phi_{i j}, 0\right)^{2}\right]^{1 / 2}
\end{aligned}
$$

and $V_{\text {ext }}\left(P_{i j}\right)$ is the extended normal velocity at point $P_{i j}$ (see the next paragraph for the computation of $\left.V_{\text {ext }}\right)$.

The numerical flux part $g_{i j}^{(2)}$ in (64) comes from the centered finite differences approximation of the second order term of the Hamilton-Jacobi equation i.e.

$$
g_{i j}^{(2)} \simeq-2 \mu P_{c}(\Omega) \mathcal{H}|\nabla \phi|\left(P_{i j}\right)=-2 \mu P_{c}(\Omega) \operatorname{div}\left(\frac{\nabla \phi}{|\nabla \phi|}\right)|\nabla \phi|\left(P_{i j}\right)
$$


The upwind part of the scheme corresponding to the discretization of the first order term of the Hamilton-Jacobi equation requires the following stability condition of CFL type :

$$
\left(\max _{U}\left|V_{\text {ext }}\right|\right) \frac{\Delta t}{\min (\Delta x, \Delta y)} \leq \frac{1}{2 \sqrt{2}} .
$$

Moreover, since we use a centered finite differences scheme for the second order term involving the curvature, the following stability condition of Fourier type has also to be satisfied :

$$
4 \mu P_{c}(\Omega) \frac{\Delta t}{\min \left(\Delta x^{2}, \Delta y^{2}\right)} \leq 1
$$

\subsection{Extended normal velocity}

As already mentioned, the normal velocity $V_{n}$ defined by (59) has to be extended to the whole domain $U$ in order to solve the Hamilton-Jacobi equation (61). A natural way to extend $V_{n}$ off the boundary $\Gamma_{N}$ is to seek a function which is constant along the curve normal to $\Gamma_{N}$. This suggests to solve at time $t$, the following equation for $q$, up to the stationary state (see [20], [22])

$$
\begin{aligned}
q_{\tau}+S(\phi) \frac{\nabla \phi}{|\nabla \phi|} \cdot \nabla q & =0 \quad \text { in } U \times \mathbb{R}^{+} \\
q(x, y, 0) & =p(x, y, t), \quad(x, y) \in U
\end{aligned}
$$

where $p$ equals to $V_{n}$ given by (59) on the boundary $\Gamma_{N}$ and 0 elsewhere. The function $S$ is an approximation of the sign function, defined by

$$
S(d)=\frac{d}{\sqrt{d^{2}+|\nabla d|^{2} \varepsilon^{2}}} \quad \text { with } \varepsilon=\min (\Delta x, \Delta y) .
$$

Another choices are possible for the approximate sign function (see [22] for details). The extended normal velocity $V_{\text {ext }}$ is given by the stationary state reached by the solution $q$ of (69),(70) as the time $\tau$ goes to $+\infty$.

At each iteration $k$ of the scheme (62)-(66), we compute the extended normal velocity as the approximate stationary solution of (69),(70). We compute $q_{i j}^{m} \simeq q\left(P_{i j}, t^{m}\right)$ from the following upwind discretization of (69) :

$$
\begin{aligned}
& q_{i j}^{m+1}=q_{i j}^{m}-\Delta \tau\left[\max \left(s_{i j} n_{i j}^{x}, 0\right) D_{x}^{-} q_{i j}^{m}+\min \left(s_{i j} n_{i j}^{x}, 0\right) D_{x}^{+} q_{i j}^{m}\right. \\
& \left.+\max \left(s_{i j} n_{i j}^{y}, 0\right) D_{y}^{-} q_{i j}^{m}+\min \left(s_{i j} n_{i j}^{y}, 0\right) D_{y}^{+} q_{i j}^{m}\right],
\end{aligned}
$$

where $s_{i j}=S\left(\phi_{i j}^{m}\right)$ and $\Delta \tau$ is a time step such that $t^{m}=m \Delta \tau$. We use central differences to compute the approximation $n_{i j}$ of the unit normal vector $n=\left(n^{x}, n^{y}\right)=\left(\partial_{x} \phi /|\nabla \phi|, \partial_{y} \phi /|\nabla \phi|\right)$ at node $P_{i j}$. The initial value $q^{0}$ is equal to $V_{n}$ on the grid points with a distance to the interface less than $\min (\Delta x, \Delta y)$ and equals zero elsewhere.

\subsection{Reinitialization to a distance function}

For numerical accuracy, the solution of the level set equation (54) shouldn't be neither too steep nor to flat. This is the case if $\phi$ is the distance function since we have in that case $|\nabla \phi|=1$. But even if we start with a distance function for the initial data $\phi_{0}$, the solution $\phi$ of the level set equation (54) does not remain a distance function. We perform a reinitialization of $\phi$ at time $t$ by solving the solution $\varphi=\varphi(x, y, \tau)$ of the following equation, up to the stationary state (see [22])

$$
\begin{aligned}
\varphi_{\tau}+S(\phi)(|\nabla \varphi|-1) & =0 \quad \text { in } U \times \mathbb{R}^{+}, \\
\varphi(x, y, 0) & =\phi(x, y, t), \quad(x, y) \in U .
\end{aligned}
$$

The function $S$ is the approximate sign function given by (71). 


\section{Numerical results}

In all the numerical tests, we choose $U=(0,1) \times(0,1)$. In the first example (see Figure 4), the topological derivative is used to create holes. In this example, the Hamilton-Jacobi equation is solved on a $51 \times 51$ grid. The data are

$$
\begin{aligned}
& f(x, y)=10 \sin ^{2}(4 \pi x), \text { for }(x, y) \in U=(0,1) \times(0,1), \\
& \lambda=0.5, \mu=0,
\end{aligned}
$$

and the initial domain $\Omega_{0}$ is the entire domain $U$ i.e. there is no hole in the initial domain. We denote by $\Omega_{1}$ the optimal domain obtained in this first example. The value of the functional $J$ in $\Omega_{1}$ is

$$
J\left(\Omega_{1}\right)=0.282319 .
$$

In the second example (see Figure 5), the topological derivative is not used but only the shape gradient is used for comparison with the previous calculation. In that case, it is therefore necessary to start with a domain with a lot of holes in order to find a domain with a proper topology. In this example, the initial domain is a square with 21 regular holes (see Figure 5) and the data $f, \lambda$ and $\mu$ are the same as in the first example.. In addition, the Hamilton-Jacobi equation is solved on a $101 \times 101$ grid. In the second example, the algorithm converges to a local maximum which is lower than the one obtained by using the topological derivative (the first example). Indeed, if we denote by $\Omega_{2}$ the optimal domain for this second example, we get

$$
J\left(\Omega_{2}\right)=0.2640695<J\left(\Omega_{1}\right)=0.282319 .
$$

Thus $\Omega_{1}$ allows an increase of $6.5 \%$ for the value of the functional $J$ compared to $\Omega_{2}$. Notice that even without topological derivative, the number of connected components of the complementary set of $\Omega$ can increase as can be seen on Figure 5 . This is not due to the creation of a hole but due to the splitting of a connected component into two connected components. This is an unusual situation, which should be related to another concept of topological derivative, called the external topological derivative (see [19]).

Additional tests have been performed with $f(x, y)=10 x+y$. In numerical tests depicted on Figure 6 and Figure 7 , there is no perimeter constraint $(\mu=0)$. We choose $\lambda=1$ on Figure 6 and $\lambda=1.7$ on Figure 7. Small or big oscillations may occur, depending on the value of the weight $\lambda$ of the volume constraint. On Figure 6 (with $\lambda=1$ ), small oscillations occur whereas big oscillations are observed on Figure 7 (with $\lambda=1.7$ ). It means that we have no optimal solution for the optimization problem since the perimeter goes to infinity. Finally, Figure 8 shows the smoothing effect of the perimeter constraint for the same source function. In this last example, we have chosen $\lambda=1.7$ and $\mu=0.4$.

\section{Appendix : The topological derivative with Neumann conditions on the hole}

We present the results which are used in Section 5 in order to determine the topological derivative of the energy functional. The asymptotic expansion of the energy is obtained with a domain decomposition technique proposed in [27], [12]. This technique can be described in the following way. The actual domain is divided into two parts $\Omega_{\rho}=\Omega_{R} \cup \Gamma_{R} \cup C(R, \rho)$ (see Figure 2). In the ring $C(R, \rho)$ the singular perturbation of the domain is located, the moving part of its boundary $\Gamma_{\rho}$ for $\rho>0$ is the small parameter. On the other part of its boundary $\Gamma_{R}$ the Steklov-Poincare operator is defined and the asymptotics of the operator are determined in function of the parameter $\rho$. The second domain $\Omega_{R}$ of the decomposition depends only on the parameter $\rho>0$ by the nonlocal boundary conditions prescribed in terms of the Steklov-Poincaré operator $A_{\rho}$, so we have the regular perturbation of the boundary conditions for the boundary value problem in $\Omega_{R}$. 

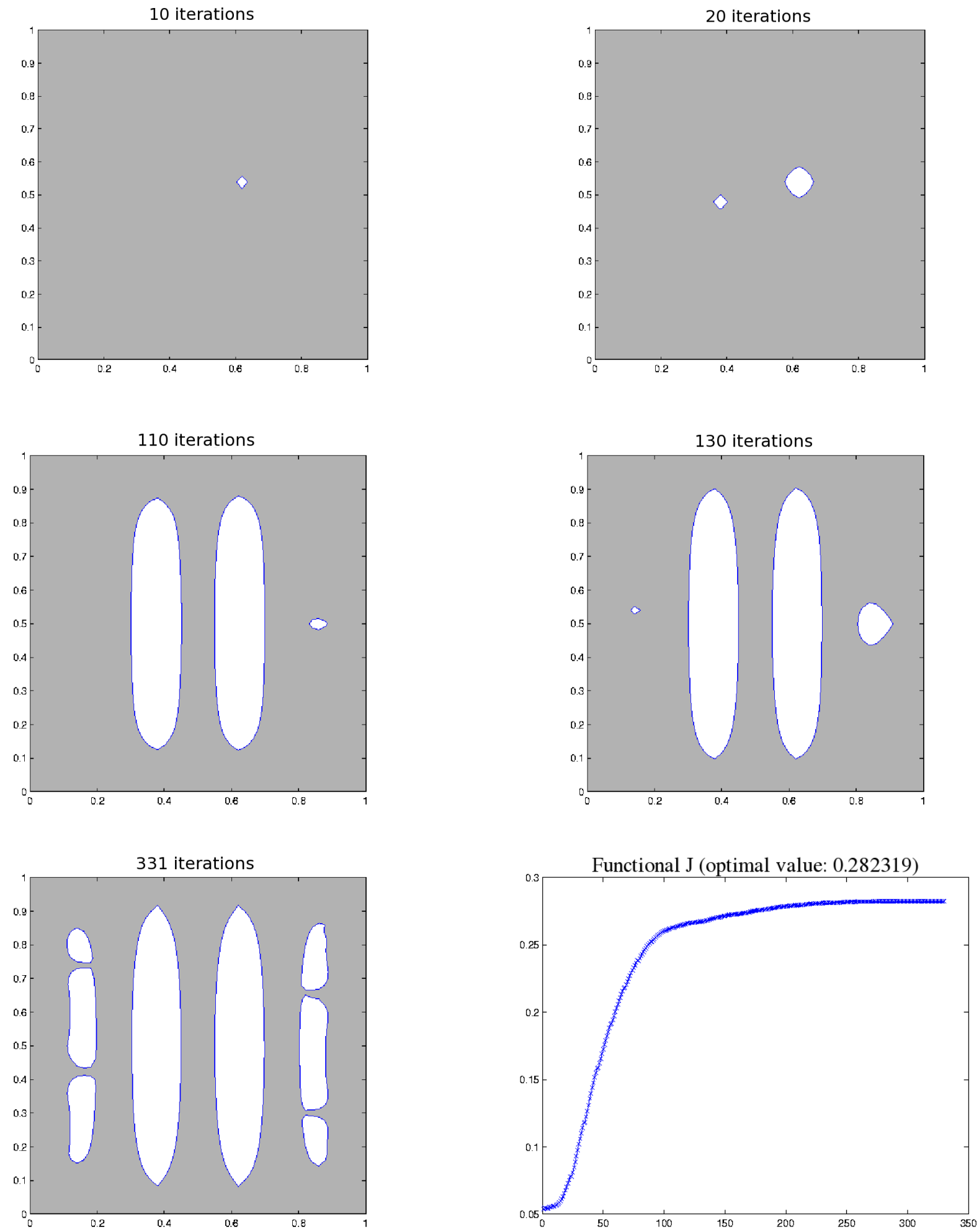

FIG. 4 - Shape optimization with topological derivative. The initial domain is without any holes $\left(f(x, y)=10 \sin ^{2}(4 \pi x), \lambda=0.5, \mu=0\right)$. 

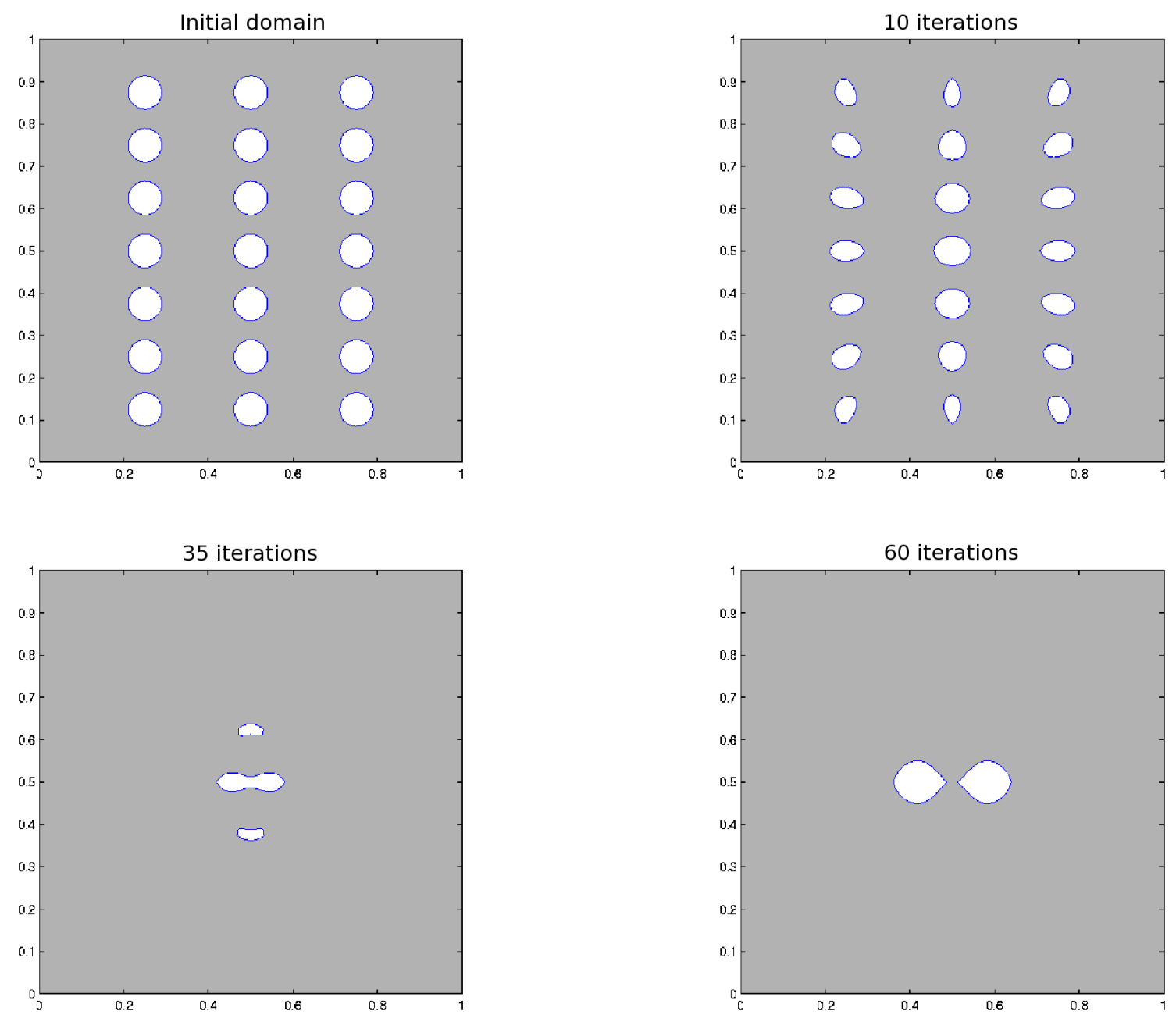

331 iterations
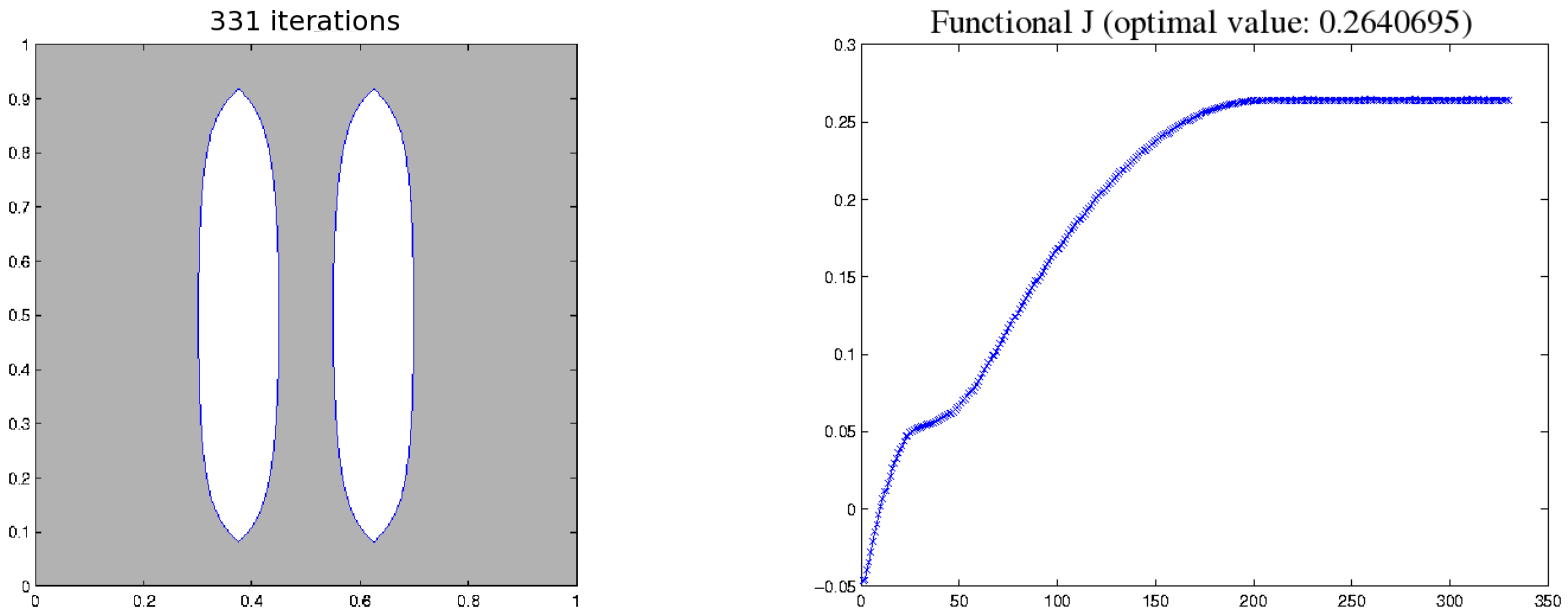

FIG. 5 - Shape optimization without the topological derivative. The initial domain is composed of 21 holes $\left(f(x, y)=10 \sin ^{2}(4 \pi x), \lambda=0.5, \mu=0\right)$. 

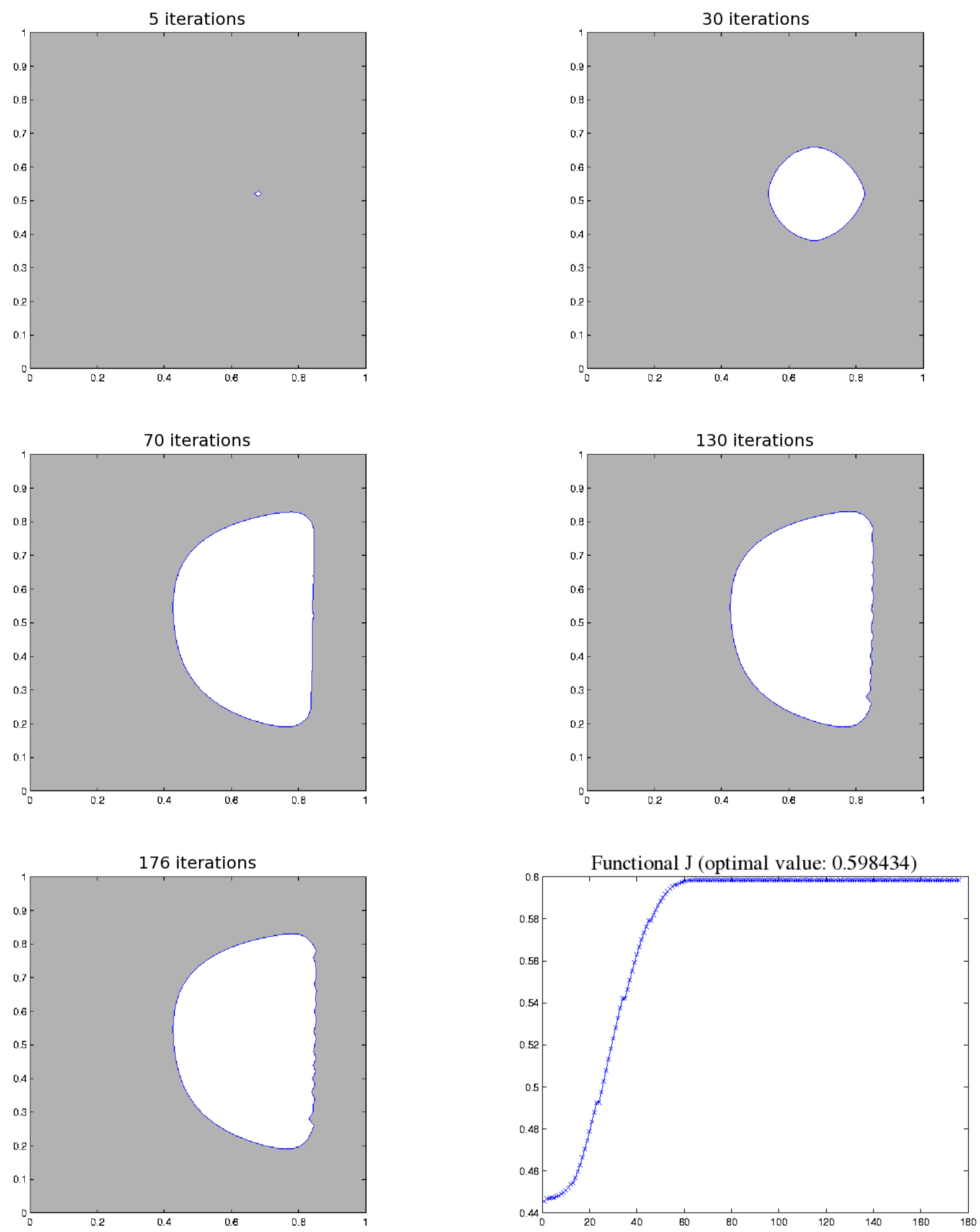

FIG. 6 - Small oscillations without any perimeter constraint $(f(x, y)=10 x+y, \lambda=1, \mu=0)$. Optimal value : $J(\Omega)=0.598434$ 

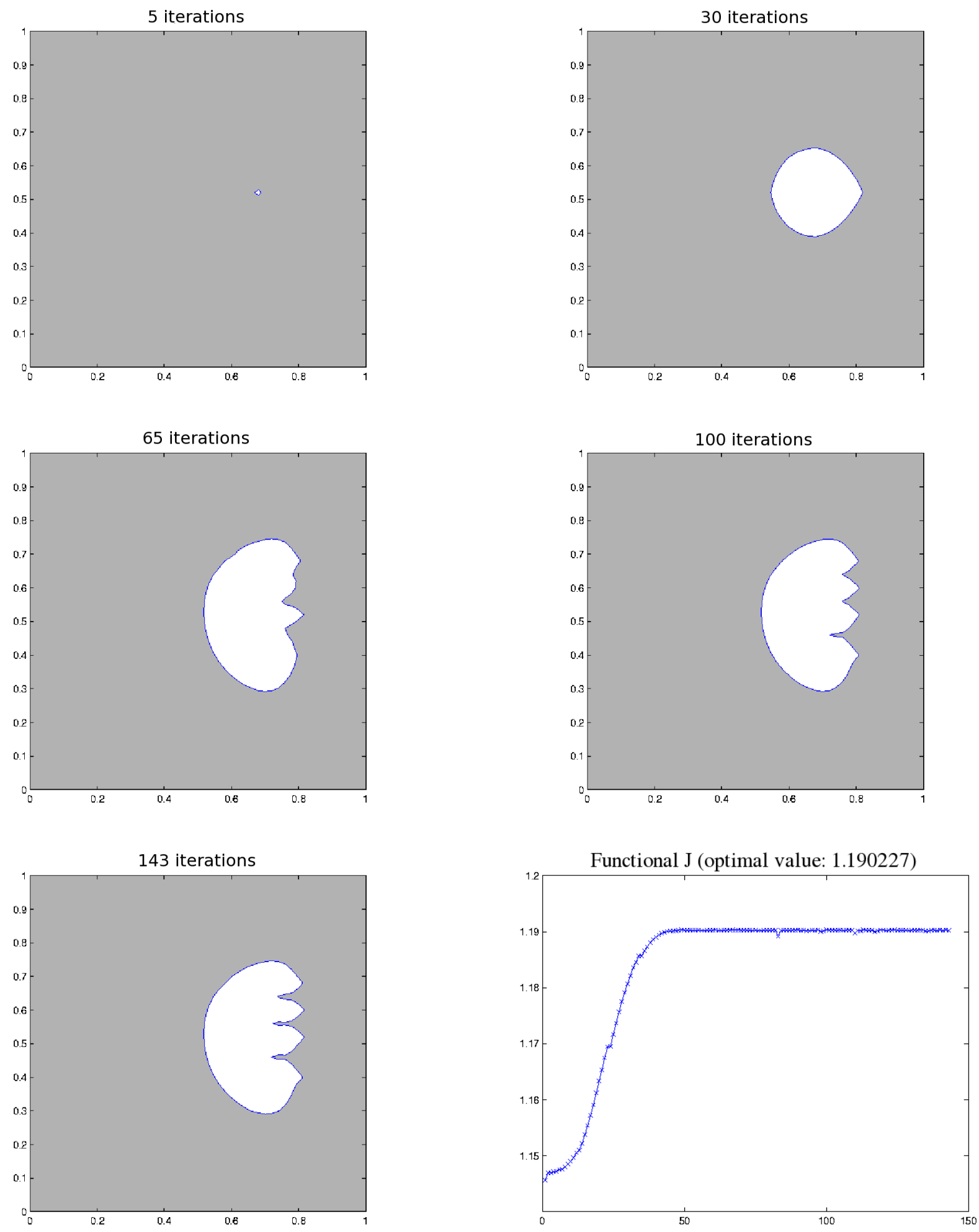

FIG. 7 - Strong oscillations without any perimeter constraint $(f(x, y)=10 x+y, \lambda=1.7, \mu=0)$. Optimal value : $J(\Omega)=1.190227$ 

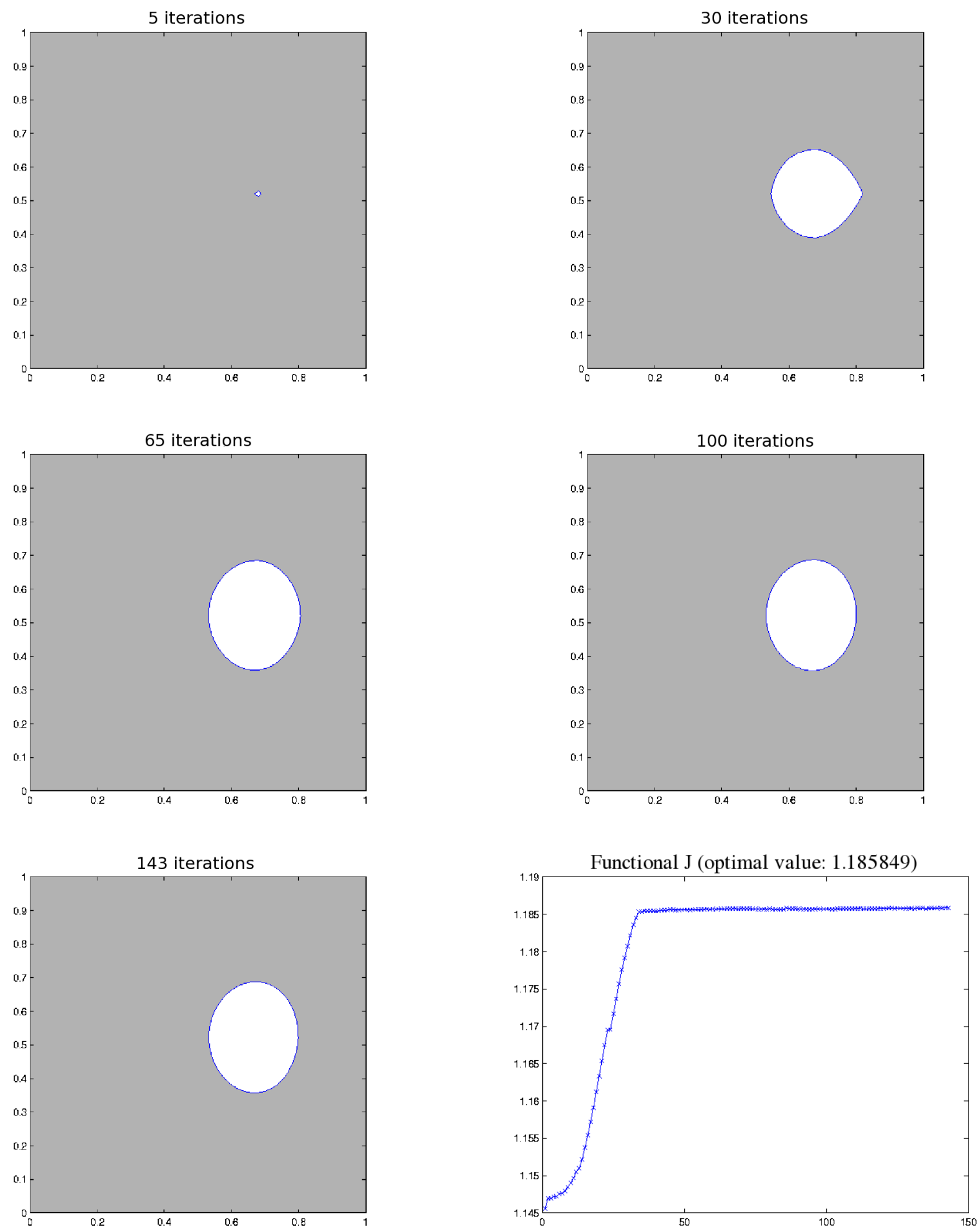

FIG. 8 - Smoothing effect of the perimeter constraint $(f=10 x+y, \lambda=1.7, \mu=0.4)$. Optimal value : $J(\Omega)=1.185849$ 
Now we give in the next subsections, the asymptotic expansions of the energy terms $E_{\rho}^{(1)}(v)$, $E_{\rho}^{(2)}(f)$ and $E\left(\Omega_{\rho}, u_{\rho}\right)$ appearing in relations (42),(43). For the sake of simplicity, we assume in what follows that $x_{0}=0$.

\subsection{Asymptotic expansion of $E_{\rho}^{(1)}(v)$}

For any function $v$ in $H^{\frac{1}{2}}\left(\Gamma_{R}\right)$, let us consider the unique solution $w_{\rho}=w_{\rho}(v)$ of the following problem (see (39))

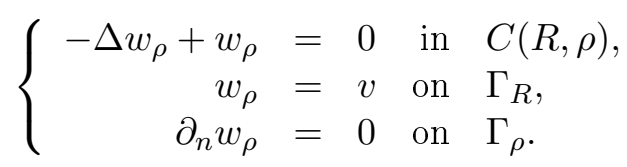

The Steklov-Poincaré operator $A_{\rho}$ is defined by

$$
\begin{aligned}
A_{\rho}: H^{\frac{1}{2}}\left(\Gamma_{R}\right) & \rightarrow H^{-\frac{1}{2}}\left(\Gamma_{R}\right) \\
v & \mapsto \partial_{n} w_{\rho}
\end{aligned}
$$

We can decompose $v \in H^{\frac{1}{2}}\left(\Gamma_{R}\right)$ in the form of Fourier series with the polar coordinates $(r, \theta)$ at the origin :

$$
v(\theta)=\frac{1}{2} a_{0}+\sum_{k=1}^{\infty}\left(a_{k} \sin (k \theta)+b_{k} \cos (k \theta)\right)
$$

and the coefficients satisfy :

$$
\sum_{k=1}^{\infty} \sqrt{1+k^{2}}\left(a_{k}^{2}+b_{k}^{2}\right) \leq M,
$$

where $M$ is a constant depending only on $R$. This implies two important properties :

$$
\sum_{k=1}^{\infty}\left(a_{k}^{2}+b_{k}^{2}\right) \leq M, \quad \sum_{k=1}^{\infty} k\left(a_{k}^{2}+b_{k}^{2}\right) \leq M .
$$

We are interested in the asymptotic expansion with respect to $\rho$ of the energy term

$$
E_{\rho}^{(1)}(v)=\int_{C(R, \rho)}\left(\left|\nabla w_{\rho}\right|^{2}+w_{\rho}^{2}\right) d x
$$

where $w_{\rho}=w_{\rho}(v)$ is the solution of (75). We define the energy functional

$$
E^{(1)}(v)=\int_{B_{R}}\left(|\nabla w|^{2}+w^{2}\right) d x
$$

where $w$ is the solution of

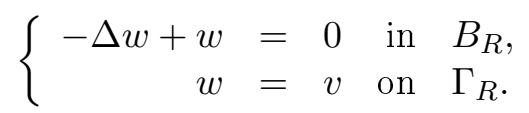

We have the following expansion result for the energy $E_{\rho}^{(1)}(v)$. For the proof, we refer to [11] or [7].

Theorem 3 There exists a constant $M>0$ independant of $\rho$ such that

$$
E_{\rho}^{(1)}(v)=E^{(1)}(v)-\left(\frac{\pi\left(a_{1}^{2}+b_{1}^{2}\right)}{2 I_{1}(R)^{2}}+\frac{\pi a_{0}^{2}}{4 I_{0}(R)^{2}}\right) \rho^{2}+o\left(\rho^{2}\right)
$$

uniformly on bounded subsets of $H^{1}\left(\Omega_{R}\right)$. The functions $I_{k}$ for $k \geq 0$, are Bessel functions of first kind defined by $I_{k}(r)=\sum_{m=0}^{\infty} \frac{\left(\frac{r}{2}\right)^{k+2 m}}{m !(k+m) !}$. 


\subsection{Asymptotic expansion of $E_{\rho}^{(2)}(f)$}

We are now interested in the expansion with respect to $\rho$ of the energy term

$$
E_{\rho}^{(2)}(f)=-\int_{C(R, \rho)}\left(\left|\nabla y_{\rho}\right|^{2}+y_{\rho}^{2}\right) d x
$$

where $y_{\rho}$ is the solution of the following problem (see (40)) :

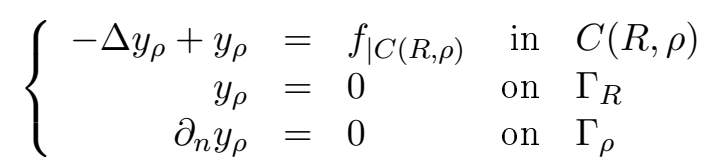

with $f \in C^{\infty}\left(\mathbb{R}^{2}\right)$. We also need to define the energy term (corresponding to $\rho=0$ in (81) and $(82))$

$$
E^{(2)}(f)=-\int_{B_{R}}\left(|\nabla y|^{2}+y^{2}\right) d x
$$

where $y$ is the solution of

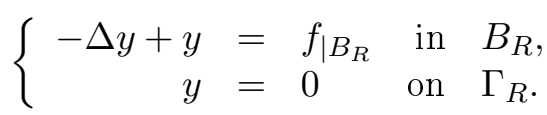

In order to get the asymptotic expansion of $E_{\rho}^{2}(f)$, we need to study the function

$$
f_{\mid C(R, \rho)} \mapsto \partial_{n} y_{\rho \mid \Gamma_{R}}=g_{\rho} .
$$

We would like to obtain an expansion of $g_{\rho}$ with respect to $\rho$. To this end, we make use of the Fourier expansion of $f$ :

$$
f(r, \theta)=\frac{1}{2} \tilde{a}_{0}(r)+\sum_{k=1}^{\infty}\left(\tilde{a}_{k}(r) \sin (k \theta)+\tilde{b}_{k}(r) \cos (k \theta)\right) .
$$

We have the following theorem (we refer to [11] or [7] for the proof) :

Theorem 4 The function $g_{\rho}$ admits the expansion

$$
g_{\rho}=g_{0}-\left(\frac{h_{0}^{a}(R)-\tilde{a}_{0}(0)}{4 R I_{0}(R)}\right) \rho^{2}-\left(\frac{h_{1}^{a}(R)}{2 R I_{1}(R)} \sin \theta+\frac{h_{1}^{b}(R)}{2 R I_{1}(R)} \cos \theta\right) \rho^{2}+o\left(\rho^{2}\right) .
$$

where $h_{i}^{a}(R), i=0,1$ are defined by

$$
h_{i}^{a}(R)=-\frac{K_{i}(R)}{I_{i}(R)} \int_{0}^{R} t \tilde{a}_{i}(t) I_{i}(t) d t+\int_{0}^{R} t \tilde{a}_{i}(t) K_{i}(t) d t
$$

and $h_{1}^{b}(R)$ is obtained from $h_{1}^{a}(R)$ by substituing $\tilde{b}_{1}$ to $\tilde{a}_{1}$ in (86). In (86), $K_{i}$ are Bessel functions of second kind [31].

From the previous result, we can deduce the asymptotic expansion for $E_{\rho}^{2}(f)$ (see [11],[7]) :

Theorem 5 There exists a positive constant $M$ independant of $\rho$ such that

$$
E_{\rho}^{(2)}(f)=E^{(2)}(f)-\frac{\pi h_{0}^{a}(R)^{2}}{4} \rho^{2}-\frac{\pi\left(h_{1}^{a}(R)^{2}+h_{1}^{b}(R)^{2}\right)}{2} \rho^{2}+o\left(\rho^{2}\right) .
$$




\subsection{Asymptotic expansion of $E\left(\Omega_{\rho}, u_{\rho}\right)$}

Recall that the energy term $E_{\rho}=E\left(\Omega_{\rho}, u_{\rho}\right)$ is given by (see (42)) :

$$
E_{\rho}=-\frac{1}{2} \int_{\Omega_{R}}\left(\left|\nabla u_{\rho}^{R}\right|^{2}+\left(u_{\rho}^{R}\right)^{2}\right) d x-\frac{1}{2} E_{\rho}^{(1)}(v)+\frac{1}{2} E_{\rho}^{(2)}(f),
$$

where $u_{\rho}^{R}$ is the solution of (37). Using Theorem 3 and Theorem 5 , we obtain

$$
\begin{aligned}
E_{\rho}-E_{0}= & \int_{\Gamma_{R}} A_{\rho}\left(u_{\rho}^{R}\right) u_{0}^{R} d \sigma-\int_{\Gamma_{R}} A_{0}\left(u_{\rho}^{R}\right) u_{0}^{R} d \sigma \\
& -\frac{\pi\left(h_{0}^{a}(R)^{2}-2 \tilde{a}_{0}(0) h_{0}^{a}(R)\right)}{8} \rho^{2}-\frac{\pi\left(h_{1}^{a}(R)^{2}+h_{1}^{b}(R)^{2}\right)}{4} \rho^{2} d \sigma \\
& +\left(\frac{\pi\left(a_{1}^{2}+b_{1}^{2}\right)}{4 I_{1}(R)^{2}}+\frac{\pi a_{0}^{2}}{8 I_{0}(R)^{2}}\right) \rho^{2}+\int_{\Gamma_{R}} u_{0}^{R} \partial_{n}\left(y_{\rho}-y_{0}\right) d \sigma+o\left(\rho^{2}\right) .
\end{aligned}
$$

It can be proved that

$$
u_{\rho}^{R}-u_{0}^{R}=o\left(\rho^{2}\right), \quad \partial_{n}\left(u_{\rho}^{R}-u_{0}^{R}\right)=o\left(\rho^{2}\right),
$$

and we deduce that

$$
\begin{aligned}
\int_{\Gamma_{R}} A_{\rho}\left(u_{\rho}^{R}\right) u_{0}^{R} d \sigma-\int_{\Gamma_{R}} A_{0}\left(u_{\rho}^{R}\right) u_{0}^{R} d \sigma & =\int_{\Gamma_{R}}\left(A_{\rho}-A_{0}\right)\left(u_{0}^{R}\right) u_{0}^{R} d \sigma+o\left(\rho^{2}\right) \\
& =\int_{\Gamma_{R}} w\left(u_{0}^{R}\right) \partial_{n} z_{\rho}\left(u_{0}^{R}\right) d \sigma+o\left(\rho^{2}\right) \\
& =-\frac{\pi a_{0}^{2}}{4 I_{0}(R)^{2}} \rho^{2}-\frac{\pi\left(a_{1}^{2}+b_{1}^{2}\right)}{2 I_{1}(R)^{2}} \rho^{2}+o\left(\rho^{2}\right) .
\end{aligned}
$$

Finally, we can calculate the last term of (87), thanks to Theorem 3 :

$$
\begin{aligned}
\int_{\Gamma_{R}} u_{0}^{R} \partial_{n}\left(y_{\rho}-y_{0}\right) d \sigma= & \int_{\Gamma_{R}} u_{0}^{R}\left(g_{\rho}-g_{0}\right) d \sigma \\
= & -\pi\left(\frac{a_{0}(R) h_{0}^{a}(R)-\tilde{a}_{0}(0) a_{0}(R)}{4 I_{0}(R)}+\frac{a_{1} h_{1}^{a}(R)}{2 I_{1}(R)}\right) \rho^{2} \\
& -\pi\left(\frac{b_{1} h_{1}^{b}(R)}{2 I_{1}(R)}\right) \rho^{2}+o\left(\rho^{2}\right) .
\end{aligned}
$$

With the previous expansions and noticing that $\tilde{a}_{0}(0)=2 f(0)$, we obtain following expansion result for the energy $E\left(\Omega_{\rho}, u_{\rho}\right)$ :

Theorem 6 There exists a positive constant $M$ independant of $\rho$ such that

$$
\begin{aligned}
E\left(\Omega_{\rho}, u_{\rho}\right)= & E(\Omega, u)-\left[\frac{a_{0}(R)^{2}}{8 I_{0}(R)^{2}}+\frac{h_{0}^{a}(R)^{2}}{8}+\frac{a_{0}(R) h_{0}^{a}(R)}{4 I_{0}(R)}\right] \pi \rho^{2} \\
& -\left[\frac{a_{1}(R)^{2}+b_{1}(R)^{2}}{4 I_{1}(R)^{2}}+\frac{a_{1}(R) h_{1}^{a}(R)}{2 I_{1}(R)}+\frac{b_{1}(R) h_{1}^{b}(R)}{2 I_{1}(R)}\right] \pi \rho^{2} \\
& -\left[\frac{h_{1}^{a}(R)^{2}}{4}+\frac{h_{1}^{b}(R)^{2}}{4}-\frac{f(0) a_{0}(R)}{2 I_{0}(R)}-\frac{f(0) h_{0}^{a}(R)}{2}\right] \pi \rho^{2}+o\left(\rho^{2}\right) .
\end{aligned}
$$

The coefficients $a_{0}, a_{1}$ and $b_{1}$ are given by

$$
a_{0}(R)=\frac{1}{\pi} \int_{0}^{2 \pi} u(R, \theta) d \theta, \quad a_{1}(R)=\frac{1}{\pi} \int_{0}^{2 \pi} u(R, \theta) \sin \theta d \theta, \quad b_{1}(R)=\frac{1}{\pi} \int_{0}^{2 \pi} u(R, \theta) \cos \theta d \theta .
$$

The functions $h_{i}^{a}(R), i=0,1$ are defined in (86). 
All the quantities between brackets in the asymptotic development (89) do not depend on $R$. It can easily be shown that

$$
u(0)=\frac{a_{0}(R)}{2 I_{0}(R)}+\frac{h_{0}^{a}(R)}{2}
$$

and then

$$
\frac{u(0)^{2}}{2}=\frac{a_{0}(R)^{2}}{8 I_{0}(R)^{2}}+\frac{h_{0}^{a}(R)^{2}}{8}+\frac{a_{0}(R) h_{0}^{a}(R)}{4 I_{0}(R)}
$$

Moreover, we have

$$
|\nabla u(0)|^{2}=\frac{a_{1}(R)^{2}+b_{1}(R)^{2}}{4 I_{1}(R)^{2}}+\frac{a_{1}(R) h_{1}^{a}(R)}{2 I_{1}(R)}+\frac{b_{1}(R) h_{1}^{b}(R)}{2 I_{1}(R)}+\frac{h_{1}^{a}(R)^{2}}{4}+\frac{h_{1}^{b}(R)^{2}}{4} .
$$

Then, we deduce a different expression of the previous asymptotic expansion, which leads actually to the usual expression for the topological derivative

$$
E\left(\Omega_{\rho}, u_{\rho}\right)=E(\Omega, u)+\left[-\frac{u(0)^{2}}{2}-|\nabla u(0)|^{2}+f(0) u(0)\right] \pi \rho^{2}+o\left(\rho^{2}\right) .
$$

Let us mention that for $i=0,1$

$$
\lim _{R \rightarrow 0} h_{i}^{a}(R)=0, \quad \lim _{R \rightarrow 0} h_{1}^{b}(R)=0 .
$$

Thus, formula (89) gives an expression of the topological derivative which requires the knowledge of $u$ along the boundary $\Gamma_{R}$. This can be interesting from a numerical point of view. In particular, it is possible to calculate $a_{1}(R)$ and $b_{1}(R)$ without directly computing the gradient of solution $u$.

\section{REFERENCES}

1. G. Allaire, F. De Gournay, F. Jouve, A.M. Toader, Structural optimization using topological and shape sensitivity via a level set method, Control and cybernetics (2005).

2. D. Bucur, Contrôle par rapport au domaine dans les E.D.P., Thèse de doctorat de l'école des mines de Paris, 1995.

3. D. Bucur, N. VArChon, Boundary variation for a Neumann problem, Ann. Scuola Norm.Sup.Pisa Cl.Sci., 429 (2000), 807-821.

4. D. Bucur, N. VARchon, A duality approach for the boundary variation of Neumann problems, SIAM J. Math Anal. Vol. 34 (2002), No.2,pp. 460-477

5. M.C. Delfour, J.-P. Zolesio, Shapes and Geometries, Advances in Design and Control. Society for Industrial and Applied Mathematics (SIAM), Philadelphia, PA, 2001.

6. G. Fremiot, Structure de la semi-dérivée eulérienne dans le cas de domaines fissurés et quelques applications, PhD Thesis of University Henri Poincaré-Nancy 1, 2000.

7. P. Fulmanski, A. Laurain, J.-F. Scheid, J. SokoŁowski, A level set method in shape and topology optimization for variational inequalities, Les prépublications de l'Institut Élie Cartan No. 32/2006.

8. A. Henrot, M. Pierre, Variation et optimisation de formes : une analyse géométrique, No 48 de Mathématiques et Applications, Springer , 2005.

9. L. Jackowska, J. SokoŁowski, A. Żochowski, A. Henrot, On numerical solution of shape inverse problems, Computational Optimization and Applications, Vol. 23, no. 2, 2002, pp. 231-255. 
10. A. L. Jackowska, J. SokoŁowski, A. Żochowski, Topological optimization and inverse problems, Computer Assisted Mechanics and Engineering Sciences, Vol. 10, no. 2, 2003, pp. $163-176$.

11. A. LAURAin, singularly perturbed domains in shape optimization, phD. Thesis, 2006, Université de Nancy.

12. M. Masmoudi, The topological asymptotic, in : $\mathrm{H} ;$ Kawarada, J.Periaux (Eds.), Computationnal Methods for Control Applications, International Series GAKUTO, 2002.

13. V. Maz'Ya, S.A. Nazarov, B. PlamenevskiJ, Asymptotic theory of elliptic boundary value problems in singularly perturbed domains, Vol. 1 and 2 Basel : Birkhäuser Verlag, 2000, $435 \mathrm{p}$.

14. S.A. NAzArov, Asymptotic conditions at a point, self adjoint extensions of operators, and the method of matched asymptotic expansions, American Mathematical Society Translations (2), Vol. 198, 1999, pp. 77-125.

15. S.A. Nazarov, A.S. Slutskij, J. Sokolowski, Topological derivative of the energy functional due to formation of a thin ligament on a spatial body, Les prépublications de l'Institut Élie Cartan No. 14/2004.

16. S.A. Nazarov, J. Sokolowski, Self adjoint extensions of differential operators in application to shape optimization, Comptes Rendus Mécanique, Volume 331, Issue 10, October 2003, 667-672.

17. S.A. Nazarov, J. Sokolowski, Selfadjoint extensions for elasticity system in application to shape optimization, to appear in Bulletin of the Polish Academy of Sciences - Mathematics.

18. S.A. Nazarov, J. SokoŁowski, Asymptotic analysis of shape functionals, Journal de Mathématiques pures et appliquées, 82(2003), 125-196.

19. S.A. Nazarov, J. Sokolowski, The topological derivative of the Dirichlet integral due to formation of a thin ligament, Siberian Math. J. March - April 2004, Volume 45, Issue 2, 341-355.

20. S. Osher, R. Fedkiw, Level set methods and dynamic implicit surfaces, Springer, 2004.

21. S. Osher, J. Sethian, Fronts propagating with curvature-dependant speed : algorithrms based on Hamilton-Jacobi formulation, J. Comp. Phys. 79, pp. 12-49, 1988.

22. D. Peng, B. Merriman, S. Osher, H. Zhao, M. Kang, A PDE-based fast local level set method, J. Comp. Phys. 155, pp. 410-438 (1999).

23. J. Sethian, Level set methods, Cambridge University Press, 1996.

24. J. SokoŁowski, A. Żochowski, On the topological derivative in shape optimization, SIAM Journal on Control and Optimization, 37, Number 4 (1999), pp. 1251-1272.

25. J. SokoŁowski, A. Żochowski, Topological derivatives of shape functionals for elasticity systems, Mechanics of Structures and Machines 29(2001), pp. 333-351.

26. J. SokoŁowski, A. Żochowski, Optimality conditions for simultaneous topology and shape optimization, SIAM Journal on Control and Optimization, Vol. 42, no. 4, 2003, pp. $1198-1221$.

27. J. SokoŁowski, A. Żochowski, Topological derivatives for contact problems, Numer. Math. 102 (2005), no. 1, 145-179.

28. J. SokoŁowski, A. Żochowski, Topological derivatives for obstacle problems, Les prépublications de l'Institut Élie Cartan No. 12/2005.

29. J. Sokolowski, J.-P. Zolesio, Introduction to shape optimization, vol. 16 of Springer Series in Computationnal Mathematics, Springer Verlag,Berlin, 1992.

30. V. Sverak, On optimal shape design, J.Math.Pures Appl., 72-6 (1993), 537-551.

31. G.N. Watson, Theory of Bessel functions Cambridge : The University Press, 1944. 